\title{
Favorable Interpretations of Ambiguity and Unstable Preferences for Fairness*
}

\author{
Emily C. Haisley \\ Department of Organizational Behavior and Theory \\ Carnegie Mellon University
}

Roberto A. Weber

Department of Social \& Decision Sciences

Carnegie Mellon University

July 26,2005

\footnotetext{
* We thank Don Moore, Colin Camerer, Matthew Rabin, and Gary Charness for comments and suggestions. Also, thanks to participants at the 2003 Meetings of the Society for Judgment and Decision Making, the 2004 Economic Science Association meetings, the 2004 Stanford Institute for Theoretical Economics meetings, and seminar participants at Carnegie Mellon, UC Berkeley, Princeton, and Emory. Please direct correspondence to Emily Haisley: ech@andrew.cmu.edu; (412) 268-5037.
} 


\begin{abstract}
We show that people manipulate their valuations of ambiguous risks when doing so allows them to justify unfair behavior. In a binary dictator decision, dictators chose between a "fair" and an "unfair" choice. By choosing the unfair choice, dictators increase their own allocation, decrease the allocation to the recipient, and make the recipient's allocation dependent on a $\mathrm{p}=0.5$ lottery. More unfair allocations were made when the lottery was ambiguous than when it involved simple risk.

We posit a mechanism through which dictators reconcile self-interest with a desire to act fairly in the presence of ambiguity. We predict and find that dictators adopt a favorably-biased view of ambiguity, as evidenced by higher estimates of the expected value of ambiguous lotteries relative to comparable lotteries involving simple risk. Dictators adopt this biased perception of ambiguity despite monetary incentives for accuracy. However, this motivated favorable view of ambiguity and increased unfair behavior is extinguished when dictators are constrained by their own initial unmotivated (negative) attitudes towards ambiguity. These findings suggest that perceptions of ambiguity can be manipulated by an underlying desire to behave self-interestedly at the expense of another, and run counter to the usual finding that ambiguity is perceived as unfavorable (ambiguity aversion).
\end{abstract}


Favorable Interpretations of Ambiguity and

Unstable Preferences for Fairness

Much research aims to uncover how concerns for fairness influence decision making. Ample evidence indicates an apparent concern for the welfare of others, even at the expense of self-interest. However, people do not display an abundance of behavior that can be described as purely altruistic (i.e. behavior that prioritizes fairness above self-interest). To complicate matters, people often demonstrate inconsistent preferences for and conceptions of fairness. As a result, understanding fair behavior may be more easily tackled by addressing the question, "under what conditions will people behave fairly or unfairly?" rather than asking "how much do people value fairness?" The current study addresses the former question by proposing that fair behavior will be influenced by the degree of subjective uncertainty, or ambiguity, surrounding the consequences of one's decisions. Specifically, we test whether the degree of uncertainty regarding the consequences of one's actions for another influences the likelihood of behaving fairly. In doing so, we also test whether ambiguity - limited information about random processes, which is typically viewed as unfavorable - can be regarded favorably when it determines the consequences of one's self-interested actions for another person.

\section{Fairness and self-serving biases}

Economic actors often appear to value fairness as they would any consumption good. People appear willing to sacrifice monetary gain to "purchase" fairness. They engage in costly altruistic behavior, such as sharing money in dictator games and donating to public goods (Dawes \& Thaler, 1988; Forsythe, Horowitz, Savin, \& Sefton, 1994), and are willing to pay to punish those who do not behave fairly (Fehr, Fischbacher, \& Gaechter, 2002; Kahneman, 
Knetsch, \& Thaler, 1986). Most people's behavior reveals an apparent concern for fairness, raising the possibility that such behavior reflects a stable and consistent preference for fair outcomes over unfair ones. Several behavioral economic models (e.g., Fehr \& Schmidt, 1999; Bolton \& Ockenfels, 2000) account for such a "preference" by incorporating preferences for fair outcomes directly into utility functions.

However, other research demonstrates that apparent preferences for fairness vary depending on the context and on factors that should not influence a stable preference. For instance, Schweitzer \& Hsee (2002) demonstrated that people show decreased concerns for fairness under greater uncertainty - specifically, subjects give more deceitful advice concerning an unknown value when the range of their private information about the true value is wide compared to when it is narrow. Oberholzer-Gee and Eichenberger (2003) found that when subjects are given the option of playing an unattractive lottery instead of playing the dictator game, many choose to opt out of the dictator game to play the lottery, even though few find the lottery attractive in a control decision. Dana et al. (2004) found that dictators prefer to remain uninformed about the consequences of their decisions for the recipient - even when this information is freely available - in order to behave self-interestedly.

These findings suggest that when subjects are faced with tradeoffs between self-interest and fairness, uncertainty or ignorance about outcomes may encourage unfair behavior. Unfair behavior may be easier to justify when there is greater uncertainty about outcomes. It is possible that greater uncertainty affords more flexible perceptions of the fairness of an action or an outcome, facilitating justifications for self-interested behavior. For example, when there is uncertainty about the consequences of one's decisions for another, the desire to engage in selfinterested behavior may inflate subjective perceptions of the likelihood of favorable 
consequences of selfish decisions for others. Previous research indicates that when people form perceptions about states of the world or about what is fair, they tend to be biased in the direction of self-interest, a finding termed "self-serving bias" (Babcock, Loewenstein, Issacharoff, \& Camerer, 1995; Diekmann, Samuels, Ross, \& Bazerman, 1997; Messick \& Sentis, 1979). Kunda (1990) proposed that such biased perception and reasoning occur when self-interests activate "motivated" cognitive processing, allowing people to justify desired conclusions with an "illusion of objectivity."

\section{Ambiguity and self-serving biases}

The current study examines the negative relationship between uncertainty and fair behavior and directly explores whether favorably-biased perceptions about uncertainty underlie this relationship. Based on the above work, we expect that increasing uncertainty about decision consequences will produce more self-interested behavior - even if the underlying decision is unchanged. Further, we propose a specific mechanism for such a relationship between uncertainty and self-interested behavior: a biased perception of uncertainty, motivated by and resulting from a self-serving desire to justify unfair choices.

Unlike previous research, the degree of uncertainty in our experiment is objectively unchanged. We only manipulate uncertainty through the distinction between simple risk and ambiguity. Simple risk applies when the underlying probability distribution is known and specific probabilities can be easily associated with each possible outcome. Ambiguity increases subjective uncertainty by decreasing the knowledge of the precise probability distribution that determines outcomes (Curley, Yates, \& Abrams, 1986; Fox \& Tversky, 1995; Keren \& 
Gerritsen, 1999). In an ambiguous situation, the precise probabilities underlying random processes are less transparent.

We implement the distinction between simple risk and ambiguity in a straightforward manner (cf. Sarin \& Weber 1993). Under simple risk, subjects face a lottery in which a chip is drawn from a bag containing precisely 10 red and 10 blue chips. Under ambiguity, the bag contains unknown, and randomly-determined, numbers of red and blue chips, totaling 20 total chips. In both cases, the subject specifies which color corresponds to a "win."

Although the ambiguous lottery described above might appear subjectively more uncertain than the lottery involving simple risk, the two lotteries are normatively identical. The probability of winning the lottery is 0.5 in both cases. However, people regularly display preferences for simple risk over ambiguity (i.e., "ambiguity aversion”). Furthermore, people will often pay an ambiguity premium to avoid the ambiguous lottery. This phenomenon has been demonstrated in numerous settings, such as laboratory choice experiments (e.g. Curley et al., 1986), market experiments (Sarin \& Weber, 1993), and in contextualized decisions (e.g. Ho, Keller, \& Keltyka, 2002). Ambiguity aversion is referred to as one of the most prominent violations of expected utility theory (Camerer \& Weber, 1992).

An interesting feature of ambiguity aversion is that people are ambiguity averse for gambling on both gains and losses at moderate to high probability levels (Curley \& Yates, 1985). The implication of this finding is that although ambiguity is viewed as unfavorable in both cases, it may be modeled as a decrease in subjective probability of outcomes involving gains and an increase in subjective probability of outcomes involving loses (Einhorn \& Hogarth, 1986; Sarin \& Wakker, 1998). In other words, people show preferences consistent with the belief that the chances of an outcome arising in a lottery decrease with ambiguity if the outcome is favorable 
and the belief that the chances of an outcome arising increase with ambiguity if the outcome is unfavorable. Additionally, subjects show preferences consistent with the belief that ambiguity is favorable when the probability of winning is small (less than $\mathrm{p}=0.25$ ) or when the probability of losing is high (greater than $\mathrm{p}=0.75)$ (Curley \& Yates 1989).

Taken together, these results indicate that people generally perceive ambiguity as unfavorable when choosing among risky options. However, the influence of ambiguity on the perceived subjective probability of events can either be positive (increased subjective probability in the case of losses) or negative (decreased subjective probability in the case of gains).

Moreover, ambiguity can be perceived as favorable or unfavorable depending on the probability range. Thus, the effects of ambiguity on perceptions of subjective probability are not constant, but instead vary depending on the decision context.

This flexibility of attitudes towards ambiguity coupled with the power of motivations to influence cognitive processes may result in favorable attitudes towards ambiguity in situations where there is a strong motivation for such a view. In particular, people who want to behave self-interestedly, but also want to believe they are behaving fairly, may find it easier to justify self-interested behavior when the consequences of decisions are based on ambiguity instead of simple risk. Specifically, we propose that a self-serving bias will lead people to manipulate their beliefs about subjective probability distributions of ambiguious risks to distributions with more favorable consequences for another (e.g., they will tend to believe that the ambiguous lottery is likely to contain more winning chips than losing chips). This bias makes unfair payoffs appear more equitable, reducing the displeasure associated with behaving unfairly.

We test the above prediction in a variation of the dictator game. Our main hypothesis is that people will make more self-intereted, i.e. less fair, choices when the consequences for a 
recipient are ambiguous than when they involve simple risk. We also expect people to overestimate the payoff to the recipient to a greater extent when decisions are made under ambiguity than simple risk, reflecting the manipulation of subjective beliefs. Finally, we predict that people who have developed attitudes towards ambiguity consistent with ambiguity aversion in a context not involving fairness will be constrained by such attitudes and unable to subsequently manipulate their subjective beliefs in order to behave self-interestedly.

\section{Experiment and Hypotheses}

Our study consisted of two treatments, each of which addressed the relationship between ambiguity, self-interest, and motivated reasoning. In the first ("Unconstrained") treatment, we tested whether subjects with no previously defined attitudes towards ambiguity would view ambiguity favorably when it determined the consequence for another of a self-interested and unfair action. In the second ("Constrained") treatment, we tested whether requiring subjects to state their own initial, presumably negative, attitudes towards ambiguity would extinguish the motivated reasoning we expected in the first treatment.

\section{Testing the relationship between unfairness and ambiguity (Unconstrained Treatment).}

We modified the standard dictator game into a decision involving a binary choice and an uncertain outcome for the recipient. In this modified game, one participant in each pair (the dictator) chose between a "fair" allocation and an "unfair" allocation for herself and an anonymous other participant (the recipient). In the fair allocation, the payoff to the dictator and the recipient were somewhat equitable and guaranteed. In the unfair allocation the dictator increased his or her payoff while decreasing the payoff to the recipient. Additionally, in the unfair allocation the payoff to the recipient depended on a lottery with a $p=0.5$ chance of 
success (and a $\mathrm{p}=0.5$ chance of no payoff). Thus, this allocation created greater inequity even if the outcome of the lottery produced a "win" for the recipient. The following is an example of such a decision:

Please select one of the following:

You receive $\$ 2$ for certain and the person you are matched with receives $\$ 1.75$ for certain OR _ You receive $\$ \mathbf{3}$ for certain and the person you are matched with wins $\$ .50$ if a winning chip is drawn in his/her lottery

Subjects in our experiment made four such choices (see Table 1). The lottery determining the payoff to the recipient involved either simple risk or ambiguity.

The primary hypothesis of this study is that ambiguity facilitates motivated reasoning in contexts involving a choice between self-interest and fairness. We predicted that ambiguity would allow dictators to manipulate their beliefs of the subjective probability of recipients' winning lotteries. Thus we expected a positive relationship between ambiguity and selfish behavior.

Hypothesis 1a: Dictators will make more unfair allocation decisions in the ambiguity condition than in the simple risk condition.

While dictators made allocation decisions with real consequences for the recipients, the recipients simultaneously made identical hypothetical decisions (which they knew did not determine outcomes). We hypothesized that since recipients' decisions were hypothetical, they would not engage in motivated reasoning. Thus, we expected no relationship between ambiguity and unfairness for the recipients. 
Hypothesis 1b: For recipients, the number of unfair decisions made in the ambiguity condition will not differ from the number of unfair decisions made in the simple risk condition.

We propose that the mechanism underlying the relationship between unfairness and ambiguity is a biased perception of the probabilities associated with ambiguous lotteries. Dictators are motivated out of self-interest to overestimate the likelihood that the lotteries associated with unfair allocations will produce a payoff for the recipient. Thus we predicted that dictators would be more unfair in ambiguity due to inflated perceptions of the expected value of lottery allocations. To test this we elicited dictators' estimates of the expected value of their allocations to the recipient, and provided monetary incentives for accuracy.

Hypothesis 2a: Dictators will overestimate the expected value of their allocation decisions more in the ambiguous condition than in the simple risk condition.

This hypothesis runs counter to previous research on ambiguity aversion, which indicates that people typically underestimate the expected value of ambiguous lotteries, compared to simple risk lotteries, for $\mathrm{p}=0.5$ lotteries involving gains (Camerer $\&$ Weber 1992). Our prediction is that motivated reasoning will be a more powerful influence over estimates than ambiguity aversion.

Recipients also made four hypothetical dictator choices, after which they also estimated the expected value that the hypothetical recipient would have received based on the four choices they made. We predicted they would not overestimate the expected value of ambiguous lotteries.

Hypothesis $2 \mathrm{~b}$ : Recipients will not overestimate the expected value of their hypothetical allocation decisions more in the ambiguous condition than in the simple risk condition. 
Following the above estimations, we asked subjects to rate the degree of fairness of the choices they had just made and their overall perceptions of how fair they view themselves. We predicted that a favorable interpretation of ambiguity would allow subjects to view each selfish choice as less unfair under ambiguity (because of the inflated subjective probability of the recipient's lottery) than under simple risk.

\section{Hypothesis 3: Controlling for the number of unfair choices made, dictators will perceive}

their behavior and themselves as more fair under ambiguity than under simple risk.

Extinguishing the effect (Constrained Treatment). Our basic argument is that increased unfair choices under ambiguity result from a favorably-biased view of ambiguity. Essential to Kunda's (1990) description of motivated reasoning is that beliefs are selectively formed that serve one's motivations or self-interests, but that these beliefs are constrained by what is plausible and must not contradict other previously-held beliefs. Therefore, we conducted an additional treatment to test whether we could extinguish the relationship between ambiguity and unfairness by constraining subjects by their own previously determined, and presumably unfavorable, attitudes towards ambiguity ${ }^{1}$. To do so, we relied on the natural propensity of people to dislike ambiguity, as demonstrated by the robust finding of ambiguity aversion.

In the Constrained Treatment, subjects first made four choices between a lottery and the expected value of that lottery (see Table 2). Fairness was not an issue in these choices. In a between subjects design, participants made these decisions under either simple risk or ambiguity. Participants then estimated the expected value of their payoffs based on these four decisions.

\footnotetext{
${ }^{1}$ This Constrained Treatment also sheds light on the casual relationship between the adoption of a favorable view of ambiguity and unfair behavior. Overestimation of the expected value of ambiguous lotteries could represent a posthoc justification for unfair behavior. Demonstrating that the relationship between ambiguity and unfairness is extinguished by a dictator's own natural unfavorable view of ambiguity indicates that our main predicted result (hypotheses 1a and 2a), is largely due to the kind of biased perception that we posit above, rather than simple posthoc justification.
} 
Participants then stated whether they preferred playing either an ambiguous or simple risk lottery for $\$ 5$. In line with previous research on ambiguity aversion, we expected most participants to be "ambiguity averse" and prefer the simple risk lottery. We hypothesized that making a decision consistent with an unfavorably-biased view of ambiguity would make subjects unable to later adopt a favorably-biased view of ambiguity in their subsequent "dictator" choices.

\section{Hypothesis 4a: In the Constrained Treatment there will be no difference in the number of} self-interested choices by dictators in the ambiguous and simple risk conditions.

We also expected that the unfavorably-biased view of ambiguity would prevent dictators from altering their subjective beliefs about the likelihood of the lottery being a "win." Therefore, we predicted that dictators' estimates of the recipients' payoffs in the Constrained Treatment would be unaffected by ambiguity versus simple risk.

Hypothesis 4b: In the Constrained Treatment there will be no significant difference between dictators' estimates of the expected value of the allocation to the recipients in the ambiguous condition compared to the simple risk condition.

Since the four initial choices involve similar dollar amounts to those received by recipients in the modified dictator game, this treatment also serves as a control to ensure that subjects are not ambiguity seeking for these kinds of lotteries. Ensuring that subjects are not ambiguity seeking for these lotteries when fairness is not a concern precludes the possible confound that subjects might chose the unfair allocation more in ambiguity simply because they think that recipients actually prefer ambiguity for these amounts and probabilities. Therefore, for these four choices, we expect that decisions will reflect the belief that ambiguous lotteries are worth no more than simple risk lotteries (a neutral or unfavorable view of ambiguity). 
Hypothesis 5a: For the four initial choices not involving fairness, subjects will choose the ambiguous lotteries no more frequently than the simple risk lotteries.

Hypothesis 5b: For the four initial choices not involving fairness, subjects will not overestimate the value of their four choices more under ambiguity than under simple risk.

Experimental Design and Methods

\section{Participants}

Participants were recruited from a subject pool of undergraduate business students. They received extra credit in one of their business courses in exchange for participation in an hour long experiment. In addition, subjects received their cash earnings privately at the end of the experiment. For the Unconstrained Treatment, we used 33 pairs of dictators and recipients (16 under simple risk, 17 under ambiguity), while in the Constrained Treatment, we used 36 pairs (19 under simple risk, 17 under ambiguity).

\section{Procedures}

Table A1 in the appendix describes the procedures, in sequence, for each treatment. Below, we present first the general procedures for the Unconstrained Treatment and then discuss differences in the Constrained Treatment.

Lotteries. Every session involved lotteries with either known (simple risk) or unknown (ambiguity) probabilities. At the beginning of the session, subjects were informed that they would be making decisions involving lotteries. The distinction between the simple risk lottery (termed "Lottery 1") and the ambiguous lottery (termed "Lottery 2") was explained. Lotteries were implemented using bags containing red and blue poker chips (participants decided which 
color they wanted to correspond to a win). Participants were informed that the contents of Lottery 2 were determined by selecting a new random number every time the lottery was played. Subjects were then told which kind of lottery would be used in their session. ${ }^{2}$

Allocation decisions and estimates. Participants were then instructed about the first set of decisions. In the Unconstrained Treatment, this was the four dictator choices. Participants were randomly assigned to the role "A" (dictators) or "B" (recipients) and paired with a participant in the opposite role. Participants were assured that their identity and the identity of the person with whom they were paired would be anonymous. Role A participants made decisions with consequences for themselves and for their matched role B participant. Role B participants made similar decisions, but their decisions were hypothetical. A's and B's were moved to opposite sides of the room following role assignment.

\begin{tabular}{|c|c|c|c|c|}
\hline \multirow{2}{*}{$\begin{array}{c}\text { Allocation } \\
\text { Decision }\end{array}$} & \multicolumn{2}{|c|}{ Fair Choice } & \multicolumn{2}{c|}{ Unfair Choice } \\
\cline { 2 - 5 } & Dictator & Recipient & Dictator & Recipient \\
\hline 1 & $\$ 1.50$ & $\$ 1.00$ & $\$ 2.25$ & $\$ 0.50$ lottery \\
\hline 2 & $\$ 2.00$ & $\$ 1.75$ & $\$ 3.00$ & $\$ 0.50$ lottery \\
\hline 3 & $\$ 2.00$ & $\$ 1.50$ & $\$ 2.25$ & $\$ 1.00$ lottery \\
\hline 4 & $\$ 1.00$ & $\$ 0.75$ & $\$ 1.50$ & $\$ 0.50$ lottery \\
\hline
\end{tabular}

Table 1. Payoffs to the dictator and the recipient for each allocation decision.

Table 1 lists the payoff structure for each allocation decision. ${ }^{3}$ Subjects received a stack of four sheets, each containing one of the decisions in Table 1. Subjects were told to go through the sheets one by one, and to not return to previous sheets. The experimenter collected these sheets and distributed another response sheet, which asked participants to estimate the expected dollar amount that the recipient of the previous four choices would receive after the resolution of

\footnotetext{
${ }^{2}$ The rationale for describing both lotteries to each session was based on empirical evidence that ambiguity is only perceived in a comparative context (Fox \& Tversky, 1995).

${ }^{3}$ We counterbalanced the order of decisions; roughly one half of subjects received the decisions in the inverse order.
} 
the four lotteries. ${ }^{4}$ They were told that for estimates within $\$ 0.10$ of the expected value they would receive a $\$ 1$ bonus.

Fairness questionnaire. Subjects then received a brief questionnaire asking them to rate the fairness of their own choices and how fair they viewed themselves more generally. Each question asked subjects to rate their response on a 9-point scale. The five questions were:

1. How fair were your choices?

2. How fair were your choices compared to the average participant?

3. How do you think your choices will be viewed by the recipient?

4. In general, how important is being fair to you?

5. In general, do you think you are a fair person?

Constrained Treatment. The Constrained Treatment was identical to the Unconstrained Treatment, except that subjects first made decisions involving risk with no conflict between selfinterest and fairness. After the explanation of lotteries at the beginning of the experiment, subjects were told that they would each make four decisions for themselves. Table 2 lists the payoff structure for each of the four decisions, which were presented in the following format:

Please select one of the following:

_ You receive $\$ .75$ for certain

$$
O R
$$

_ You receive $\$ \mathbf{1 . 5 0}$ if a winning chip is drawn in a lottery

Subjects received a stack of sheets containing the four decisions and were instructed to go through them one at a time. After the experimenter collected the choices, subjects received a sheet asking them to estimate the expected value of their own payoff.

\footnotetext{
${ }^{4}$ We explicitly asked subjects for the "expected value." To illustrate the concept of expected value, we informed participants that for every combination of choices there is a correct estimate, based on the laws of probability, and this is the best guess one could make given that the outcomes of the lotteries are not yet known.
} 


\begin{tabular}{|c|c|c|}
\hline Decision & Choice 1 & Choice 2 \\
\hline 1 & $\$ .75$ & $\$ 1.50$ lottery \\
\hline 2 & $\$ .50$ & $\$ 1.00$ lottery \\
\hline 3 & $\$ 1.00$ & $\$ 2.00$ lottery \\
\hline 4 & $\$ .25$ & $\$ .50$ lottery \\
\hline
\end{tabular}

Table 2: Initial choices in Constrained Treatment

Subjects in the Constrained Treatment then chose which kind of lottery ( 1 or 2$)$ they would like to play for the chance to win $\$ 5$ and which color they wanted to bet on. Participants were informed that one participant would be selected at the end of the session at random to play the lottery. The Constrained Treatment then proceeded identically to the Unconstrained Treatment, beginning with random assignment to the role of A (dictator) or B (recipient) and instructions regarding the modified dictator game.

\begin{tabular}{|l|l|c|c|}
\hline & & Unconstrained & Constrained \\
\hline \multirow{2}{*}{$\begin{array}{l}\text { Real dictators } \\
\text { (A participants) }\end{array}$} & Simple risk & $2.25(56 \%)$ & $2.32(58 \%)$ \\
\cline { 2 - 4 } $\begin{array}{l}\text { Hypothetical dictators } \\
\text { (B participants) }\end{array}$ & Ambiguity & $3.06(77 \%)$ & $2.18(55 \%)$ \\
\cline { 2 - 4 } & Simple risk & $2.56(64 \%)$ & $2.11(53 \%)$ \\
\hline
\end{tabular}

Table 3. Number of unfair choices by treatment, condition, and role

Results

Table 3 presents the number of unfair choices in the four allocation decisions by condition (ambiguity vs. simple risk) and treatment (Unconstrained vs. Constrained) for both real dictators (A participants) and hypothetical dictators (B participants). The results support our main predictions regarding the frequency of unfair choices. The highest frequency of unfair choices is made by real dictators under ambiguity in the Unconstrained Treatment. To more carefully test our prediction, we consider the separate hypotheses presented earlier. 
Allocation decisions. Hypothesis 1a predicted that dictators in the Unconstrained Treatment would make more unfair choices under ambiguity than under simple risk. This was confirmed. Dictators in the simple risk condition made an average of 2.25 unfair decisions $(56 \%, \mathrm{n}=16)$, while dictators in the ambiguous condition made an average of 3.06 unfair decisions $(77 \%, \mathrm{n}=17)$. Comparing the mean number of unfair decisions of each individual (i.e., the subject is the unit of observation), we find that this difference is statistically significant $(\mathrm{t}(31)=1.87, p=.04$, one-tailed $)$.

The dictator's decision had real consequences for the recipients, meaning they were motivated to find justifications for unfair choices, such as viewing ambiguity as favorable. In contrast, the recipients' decisions were only hypothetical and thus recipients were not motivated to use ambiguity as a justification for unfair decisions. Hypothesis $1 \mathrm{~b}$ predicted that there would not be a significant difference between the numbers of unfair hypothetical decisions under ambiguity compared to those made under simple risk for the recipients. This was confirmed. In the simple risk condition recipients "hypothetical dictators" made an average of 2.56 (64\%, $\mathrm{n}=16)$ unfair decisions, while in the ambiguous condition this number was $2.41(60 \%, \mathrm{n}=17)$. This difference is not statistically significant $(\mathrm{t}(31)=0.31, p=0.76$, two-tailed $){ }^{5}$

Estimates of allocations to recipient. The degree of overestimation of the expected value of one's allocations to the recipient represents an overestimation of one's generosity. Consistent with Hypothesis 2a, dictators in the Unconstrained Treatment overestimated the expected value of their allocations to the recipient more in ambiguity than in simple risk. In the simple risk condition, dictators underestimated the expected value of their four allocations to the recipient by $\$ 0.17(\mathrm{n}=11)$, while in the ambiguous condition, dictators overestimated the value of the

\footnotetext{
${ }^{5}$ In line with Hypothesis $1 \mathrm{~b}$, there is a marginally significant interaction between ambiguity and role (real decisions or hypothetical decisions) on the number of unfair decisions ( $\beta=-.96, p=.07$, one-tailed).
} 
expected value of their allocations by $\$ 1.37(n=17)$. This comparison is represented by the two leftmost bars in Figure 1. This difference is statistically significant $(\mathrm{t}(26)=2.35, p=.01$, onetailed).

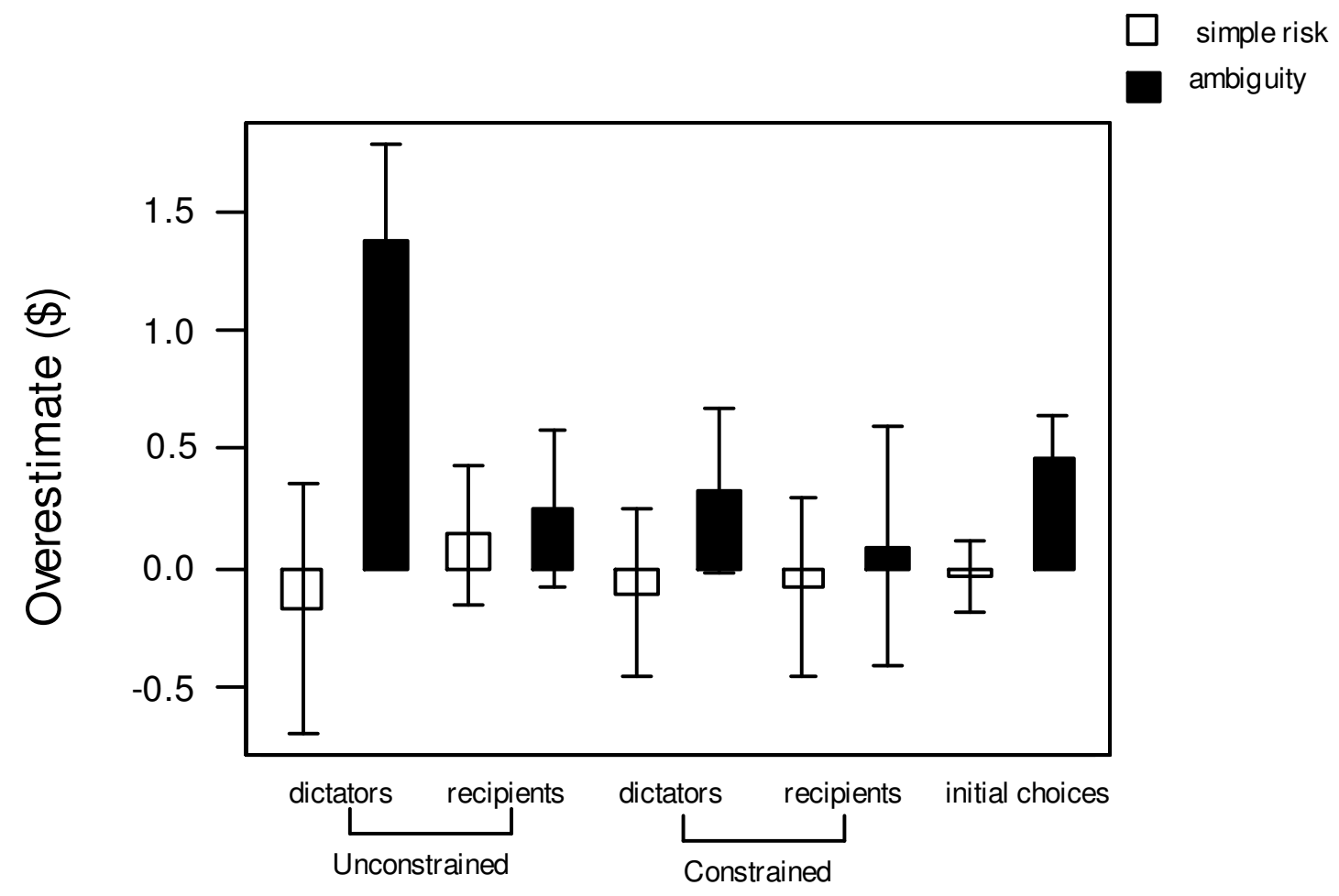

Figure 1. Degree of overestimation of expected payoff (estimate - actual)

As predicted by Hypothesis $2 \mathrm{~b}$, we found no difference in the degree of overestimation between ambiguity and simple risk for recipients' estimates of their hypothetical allocations. Under simple risk, the mean overestimate was $\$ 0.14(n=11)$, while under ambiguity it was $\$ 0.25$ 
$(\mathrm{n}=17)$ and this difference is not significant $(\mathrm{t}(26)=.23, p=.82$, two-tailed test $)$. This difference is represented by the second set of two bars in Figure $1 .^{6}$

Fairness questionnaire. After making their choices, subjects answered five questions regarding the fairness of their behavior and their own disposition to behave fairly. Hypothesis 3 predicted that their responses to these questions would reflect a belief that selfish choices made under ambiguity were less unfair that those made under simple risk.

Table 4 presents the results of regressions with responses to the five questionnaire items as dependent variables and three independent variables: number of unfair choices, a binary variable indicating the subject chose under ambiguity (1) or simple risk (0), and an interaction term between unfair choices and ambiguity. ${ }^{7}$ Hypothesis 3 predicted that the perceptions of one's own fairness would be less negatively influenced by making unfair choices under ambiguity than under simple risk (i.e., the coefficient on the interaction term would be positive).

As the results in Table 4 indicate, dictators recognize the unfairness of self-interested choices (row 1). The analysis reveals mixed support for the hypothesis that self-interested behavior under ambiguity would not be viewed as unfair as under simple risk (italicized row). Specifically, for Item 3 ambiguity appears to decrease the effect of unfair choices on rated fairness by about one-half ( $\mathrm{p}=.07$, one-tailed), while for Item 5 it appears to eliminate almost all the effect ( $\mathrm{p}=.05$, one-tailed). However, the other effects are small, and the sign of the coefficient for Item 1 is opposite of what we hypothesized. Therefore, there is only modest support for Hypothesis $3 .^{8}$

\footnotetext{
${ }^{6}$ In line with Hypothesis $2 \mathrm{~b}$, there is a significant interaction between ambiguity and role (real decisions or hypothetical decisions) on allocation estimates ( $\beta=-1.43, p=.04$, one-tailed).

${ }^{7}$ The analysis includes only subjects in the role of real dictators (A participants).

${ }^{8}$ One possible explanation for the weak result lies in the payoffs in Table 1. Note that even if the lottery produces a "win" for the recipient (which we predicted dictators would perceive as more likely under ambiguity), the outcome
} 


\begin{tabular}{|l|c|c|c|c|c|}
\hline & \multicolumn{5}{|c|}{ Dependent variable (all measured on a 9-point scale) } \\
\hline & $\begin{array}{c}\text { Item1 } \\
\text { How fair were } \\
\text { your choices? }\end{array}$ & $\begin{array}{c}\text { Item 2 } \\
\text { How fair } \\
\text { compared to avg. } \\
\text { participant? }\end{array}$ & $\begin{array}{c}\text { Item 3 } \\
\text { How will choices } \\
\text { be viewed by } \\
\text { recipient? }\end{array}$ & $\begin{array}{c}\text { Item 4 } \\
\text { How important is } \\
\text { being fair to you? }\end{array}$ & $\begin{array}{c}\text { Item 5 } \\
\text { Do you think you } \\
\text { are a fair person? }\end{array}$ \\
\hline S.I. choices & $-0.76(0.34)^{* * *}$ & $-0.70(0.20)^{* * *}$ & $-1.58(0.24)^{* * *}$ & $-0.28(0.29)$ & $-0.44(0.19)^{* *}$ \\
\hline Ambiguity & $2.76(2.02)$ & $0.09(1.16)$ & $-1.69(1.44)$ & $-1.23(1.73)$ & $-0.99(1.13)$ \\
\hline Amb. $x$ SI ch & $-0.67(0.66)$ & $0.07(0.38)$ & $0.72(0.47)^{* * a}$ & $0.24(0.57)$ & $0.64(0.37)^{* * a}$ \\
\hline Constant & $6.08(0.92)^{* * * *}$ & $6.95(0.53)^{* * * *}$ & $7.36(0.66)^{* * *}$ & $7.81(0.79)^{* * *}$ & $7.79(0.52)^{* * *}$ \\
\hline N & 33 & 33 & 33 & 33 & 33 \\
\hline R-squared & 0.280 & 0.367 & 0.625 & 0.073 & 0.213 \\
\hline
\end{tabular}

Standard errors in parentheses; ${ }^{*}-\mathrm{p}<0.1 ;^{* *}-\mathrm{p}<0.05 ;^{* * * *}-\mathrm{p}<0.01$; $^{\mathrm{a}}-$ one-tailed

Table 4. Regressions of perceptions of fairness on condition and behavior

\section{Extinguishing the effect (Constrained Treatment)}

We predicted that the relationship between ambiguity an unfair allocation decisions would be extinguished if dictators fist made a decision in which they stated their own attitudes towards ambiguity (presumably ambiguity averse) in a context not involving fairness. As expected, a majority of subjects exhibited ambiguity aversion in this decision, with 67 percent of the dictators in the ambiguous condition preferring the simple risk lottery $(n=72)$. This is significantly different from 50 percent using a binomial test $(\mathrm{p}<0.01$, one-tailed).

Allocation decisions. Subjects then made four binary dictator allocation decisions in exactly the same way as in the Unconstrained Treatment. One half of the subjects were real dictators, while the other half were the recipients who made identical hypothetical decisions. Hypothesis 4a predicted no difference between the choices made by real dictators under ambiguity and simple risk, and this is supported by the data. In the simple risk condition, dictators made an average of 2.32 unfair choices (58 percent, $n=19$ ), while under ambiguity they 
made an average of 2.18 unfair choices (54 percent, $\mathrm{n}=17$ ). This difference is not significantly different $(\mathrm{t}(36)=.27, \mathrm{p}=.78$, two-tailed $)$.

An implication of Hypothesis $4 \mathrm{a}$ is that dictators who make decisions under ambiguity in the Unconstrained Treatment should be significantly less fair (because they can adopt a favorable view of ambiguity) than dictators under ambiguity in the Constrained Treatment (who are constrained by their initial attitudes towards ambiguity). We find this is true: the average number of unfair choices in the ambiguity condition for Constrained dictators is 2.18 (54 percent), while for Unconstrained dictators it is 3.06 (77 percent). This difference is statistically significant $(\mathrm{t}(34)=2.03, \mathrm{p}=.03$, one-tailed). Subjects who were required to state an attitude towards ambiguity earlier in the experiment - in a context not involving fairness - are significantly less likely to give ambiguous lotteries to recipients. Estimates of allocations to recipient. We predicted that dictators in the Constrained Treatment would be unable to engage in motivated reasoning to view ambiguity as favorable, and that this would be reflected in their estimates of the recipient's payoff. Hypothesis $4 \mathrm{~b}$ predicted that the degree of overestimation of the recipient's payoff would be equal under ambiguity and simple risk in the Constrained Treatment. Dictators in the simple risk condition underestimated the expected value of recipients' payoffs by $\$ 0.10$ on average $(n=19)$ while dictators in the ambiguity condition overestimated the value by $\$ 0.33$ on average $(n=17)$. This comparison is presented in the middle two bars of Figure 1. This difference is not statistically significant $(\mathrm{t}(34)=.87, p=.39$, two-tailed), supporting Hypothesis 4b. Hypothesis $4 \mathrm{~b}$ is further supported by the fact that the degree of overestimation by dictators under ambiguity in the Constrained Treatment (\$0.33) is significantly lower than 
the degree of overestimation by dictators under ambiguity in the Unconstrained Treatment $(\$ 1.37)(\mathrm{t}(32)=1.96, \mathrm{p}=.03$, one-tailed $)$.

Initial choices as a control. In the Constrained Treatment, subjects started off by making four choices that did not involve allocations to another. The roles of dictator and recipient had not been explained yet, so every subject made this choice. ${ }^{9}$ In each of these choices, a subject chose between some certain amount and a lottery (see Table 2). Hypothesis 5a predicted that subjects would choose the lottery under simple risk at least as often as under ambiguity. This hypothesis is confirmed. Subjects in the simple risk condition chose the lottery on average in 2.89 out of 4 choices $(72 \%, n=38)$, while subjects in the ambiguity condition did so in $2.91(73 \%, \mathrm{n}=34)$. Since the two proportions are almost identical we can reject the possible confound that, for this level of stakes and probability, the ambiguous lotteries are actually more attractive to recipients than those involving simple risk.

Participants also estimated the expected value of their own payoffs for these decisions. Hypothesis $5 \mathrm{~b}$ predicted that the overestimation would be no greater for ambiguous lotteries than for simple risk lotteries. Counter to Hypothesis 5b, however, overestimation was slightly higher under ambiguity compared to simple risk. The value of payoffs was underestimated slightly in simple risk, by $\$ 0.04(\mathrm{n}=38)$, and overestimated in ambiguity, by $\$ 0.46(n=34)$. These estimates are presented in Figure 1 by the two rightmost bars. This difference is statistically significant $(\mathrm{t}=2.16, p=.03$, two-tailed).

While subjects overestimated their payoffs for these decisions under ambiguity, the degree of overestimation is significantly smaller than for dictators' estimates of the value of their allocations to recipients under ambiguity in the Unconstrained Treatment $(\mathrm{t}=2.41$,

\footnotetext{
${ }^{9}$ Participants had received ID numbers (1A, 1B, 2A, 2B, 3A, 3B, etc.) but had not yet been told what the letter portion of the ID represented. There are no significant differences by (subsequent) role for these initial choices.
} 
$p=.02$, two-tailed). This means that although we find some tendency to overestimate in ambiguity for choices not involving fairness, the degree of overestimation is roughly three times as large for decisions that do involve fairness, and this difference is significant.

\section{Discussion}

This study presents strong evidence in support of a positive relationship between ambiguity and unfair behavior. While most people prefer simple risk to ambiguity for themselves, we find that ambiguity becomes more attractive when it is the determinant of consequences of our selfish actions for others. That is, people view ambiguity as favorable when it allows them to view what they are doing to others as "not that unfair." We find clear evidence of such manipulation of attitudes towards ambiguity. Subjects are more likely to give low-paying lotteries to others when these lotteries involve ambiguity compared to simple risk, and they subsequently treat these lotteries as more attractive, reflected by higher estimates of their expected value. ${ }^{10}$

The results of the Constrained Treatment suggest that the adoption of a favorablybiased view of ambiguity is the cause of increased unfair behavior under ambiguity, as opposed to merely being a post-hoc justification for unfair treatment. When dictators are thwarted from adopting a favorable attitude towards ambiguity, they do not exhibit elevated unfair behavior or inflated perceptions of the value of ambiguous lotteries.

\footnotetext{
${ }^{10}$ We found additional evidence that ambiguity can be viewed favorably in contexts pitting self-interest against fairness considerations. Following the experiments, while waiting for payment calculations, subjects completed a "pilot experiment". They first made a hypothetical choice between two "fair" lotteries (roughly equal payoffs for both the subject and a recipient; e.g., \$2.00 \& \$1.75) and "unfair" lotteries (with a higher payoff for the dictator but a lower one for the recipient, e.g., \$4.00 \& \$0.25). Subjects then specified which kind of lottery (Lottery 1 or Lottery 2) they wanted to determine the payoffs resulting from the choice. We found that subjects chose the ambiguous lottery over twice as often after having chosen unfairly than if they had chosen the fair option.
} 
Almost all previous research on ambiguity indicates that people interpret ambiguity unfavorably. ${ }^{11}$ Our results indicate that how people interpret ambiguity is more complicated. In contexts where there exists a motivation to view uncertainty favorably, people will adopt a more favorable view if they are unconstrained by other factors, such as their own previous attitudes. People's overall attitudes towards ambiguity may be more accurately described by "ambiguity distortion" rather than ambiguity aversion.

Our findings are consistent with previous evidence suggesting that people will engage in "moral wriggling," seizing justifications to behave self-interestedly without explicitly appearing so to themselves or others (Dana, Weber, \& Xi Kuang, 2004; Schweitzer \& Hsee, 2002). In this paper, we show that ambiguity about decision consequences presents just such a justification. Under ambiguity, dictators are able to convince themselves that the selfinterested option is not as bad as it seems under known risk. That such motivated reasoning produces outcomes that are less fair perhaps provides evidence of subjects' true motivation. The power of motivated reasoning is evidenced by the finding that dictators give biased estimates in line with their motivations, even when they can profit by giving an unbiased estimate.

\footnotetext{
${ }^{11}$ The few exceptions include ambiguity seeking at low probabilities (<.25) (Curley \& Yates, 1989) and indifference between ambiguous and simple risk lotteries in non-comparative settings (Fox, 1995).
} 


\section{References}

Babcock, L., Loewenstein, G., Issacharoff, S., \& Camerer, C. F. (1995). Biased Judgments of Fairness in Bargaining. American Economic Review, 85(5), 1337-1343.

Camerer, C., \& Weber, M. (1992). Recent Developments in Modeling Preferences: Uncertainty and Ambiguity. Journal of Risk and Uncertainty, 5(4), 325-370.

Curley, S. P., \& Yates, F. J. (1989). An empirical evaluation of descriptive models of ambiguity reactions in choice situations. Journal of Mathematical Psychology, 33(4), 397-427.

Curley, S. P., \& Yates, J. F. (1985). The center and range of the probability interval as factors affecting ambiguity preferences. Organizational Behavior \& Human Decision Processes, $36(2), 273-287$.

Curley, S. P., Yates, J. F., \& Abrams, R. A. (1986). Psychological sources of ambiguity avoidance. Organizational Behavior \& Human Decision Processes, 38(2), 230-256.

Dana, J., Weber, R., \& Xi Kuang, J. (2004). Do People Value Being Fair or Not Being Unfair? Behavior Inconsistent with "Fairness Preferences".Unpublished manuscript.

Dawes, R., \& Thaler, R. (1988). Annomolies: Cooperation. Journal of Economic Perspectives, 2(3), 187-197.

Diekmann, K. A., Samuels, S. M., Ross, L., \& Bazerman, M. H. (1997). Self-interest and fairness in problems of resource allocation: Allocators versus recipients. Journal of Personality \& Social Psychology, 72(5), 1061-1074.

Einhorn, H. J., \& Hogarth, R. M. (1986). Decision Making under Ambiguity. Journal of Business, 59(4), S225-250.

Fehr, E., Fischbacher, U., \& Gaechter, S. (2002). Strong reciprocity, human cooperation, and the enforcement of social norms. Human Nature, 13(1), 1-25. 
Forsythe, R., Horowitz, J. L., Savin, N. E., \& Sefton, M. (1994). Fairness in Simple Bargaining Experiments. Games and Economic Behavior, 6(3), 347-369.

Fox, \& Tversky, A. (1995). Ambiguity Aversion and Comparative Ignorance. Quarterly Journal of Economics, 110(3), 585-603.

Ho, J. L. Y., Keller, L. R., \& Keltyka, P. (2002). Effects of Outcome and Probabilistic Ambiguity on Managerial Choices. Journal of Risk and Uncertainty, 24(1), 47-74.

Kahneman, D., Knetsch, J. L., \& Thaler, R. (1986). Fairness as a Constraint on Profit Seeking: Entitlements in the Market. American Economic Review, 76(4), 728-741.

Keren, G., \& Gerritsen, L. E. M. (1999). On the robustness and possible accounts of ambiguity aversion. Acta Psychologica, 103(1-2), 149-172.

Kunda, Z. (1990). The case for motivated reasoning. Psychological Bulletin, 108(3), 480-498.

Messick, D. M., \& Sentis, K. P. (1979). Fairness and preference. Journal of Experimental Social Psychology, 15(4), 418-434.

Oberholzer-Gee, F., Eichenberger, R. (2003). Fairness in extended dictator game experiments. Working Paper.

Sarin, R., \& Wakker, P. P. (1998). Revealed Likelihood and Knightian Uncertainty. Journal of Risk and Uncertainty, 16(3), 223-250.

Sarin, R. K., \& Weber, M. (1993). Effects of Ambiguity in Market Experiments. Management science, 39(5), 602.

Schweitzer, M. E., \& Hsee, C. K. (2002). Stretching the Truth: Elastic Justification and Motivated Communication of Uncertain Information. Journal of Risk and Uncertainty, $25(2), 185-201$. 
Appendix

\begin{tabular}{|c|c|}
\hline Unconstrained Treatment & Constrained Treatment \\
\hline $\begin{array}{l}\frac{\text { Lotteries }}{-\quad} \\
\text { ("Loscription of two kinds of lotteries } \\
\text { ("Lottery 1" and "Lottery 2"). } \\
\text { Subjects told which lottery would be used } \\
\text { in experiment }\end{array}$ & $\begin{array}{l}\frac{\text { Lotteries }}{-} \text { Description of two kinds of lotteries } \\
\text { ("Lottery 1" and "Lottery 2") } \\
\text { - Subjects told which lottery would be used } \\
\text { in experiment }\end{array}$ \\
\hline $\begin{array}{l}\text { Dictator game } \\
-\quad \text { Description of dictator game (Table 1) } \\
\text { - A participants make } 4 \text { real choices } \\
\text { - B participants make } 4 \text { hypothetical } \\
\text { choices }\end{array}$ & $\begin{array}{l}\text { Choices involving risk } \\
\text { - Description of choices between lotteries } \\
\text { and certainty equivalents (Table 2) } \\
\text { - } \quad \text { All participants make } 4 \text { choices }\end{array}$ \\
\hline $\begin{array}{l}\text { Estimates of expected payoff to recipient } \\
\text { based on own (real or hypothetical) choices }\end{array}$ & $\begin{array}{l}\text { Estimates of expected payoff to self based on } \\
\text { choices }\end{array}$ \\
\hline Fairness questionnaire & $\begin{array}{l}\text { Choice of Lottery } 1 \text { vs. Lottery } 2 \text { for } \$ 5 \text { (one } \\
\text { subject selected at end of experiment to play) }\end{array}$ \\
\hline Resolution of all lotteries & $\begin{array}{l}\text { Dictator game } \\
\quad \text { Description of dictator game (Table 1) } \\
\text { - A participants make } 4 \text { real choices } \\
-\quad \text { B participants make } 4 \text { hypothetical } \\
\text { choices }\end{array}$ \\
\hline $\begin{array}{l}\text { Pilot experiment while waiting for payment } \\
\text { calculation }\end{array}$ & $\begin{array}{l}\text { Estimates of expected payoff to recipient } \\
\text { based on own (real or hypothetical) choices }\end{array}$ \\
\hline & Resolution of all lotteries \\
\hline
\end{tabular}

Table A1. Sequence of procedures by treatment in experiment 1 . 